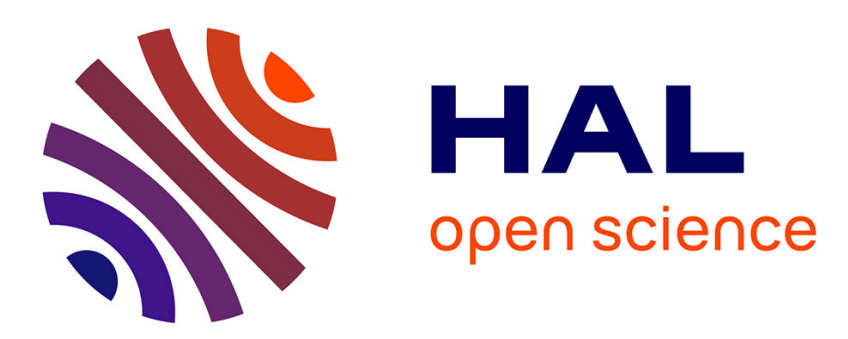

\title{
Algebraic Insight on the Concomitant Motion of 3RPS and 3PRS PKMs
}

Hassen Nigatu, Yimesker Yihun

\section{To cite this version:}

Hassen Nigatu, Yimesker Yihun. Algebraic Insight on the Concomitant Motion of 3RPS and 3PRS PKMs. Computational Kinematics Proceedings of the 7th International Workshop on Computational Kinematics, 2020, pp.242 - 252. 10.1007/978-3-030-43929-3_22 . hal-03233161

\section{HAL Id: hal-03233161 \\ https://hal.science/hal-03233161}

Submitted on 23 May 2021

HAL is a multi-disciplinary open access archive for the deposit and dissemination of scientific research documents, whether they are published or not. The documents may come from teaching and research institutions in France or abroad, or from public or private research centers.
L'archive ouverte pluridisciplinaire HAL, est destinée au dépôt et à la diffusion de documents scientifiques de niveau recherche, publiés ou non, émanant des établissements d'enseignement et de recherche français ou étrangers, des laboratoires publics ou privés. 


\title{
Algebraic Insight on the Concomitant Motion of 3RPS and 3PRS PKMs
}

\author{
Hassen Nigatu ${ }^{1}$ and Yimesker Yihun ${ }^{2(凶)}$ \\ 1 Korea Institute of Science and Technology (KIST), \\ University of Science and Technology (UST, Korea), Seoul, Korea \\ 614503@kist.re.kr, hassity@gmail.com \\ 2 Wichita State University, Wichita, USA \\ yimesker.yihun@wichita.edu
}

\begin{abstract}
This study presents an algebraic method to detect, count, and identify concomitant motions of parallel robots at the velocity level. The pose and orientation of moving platform of parallel manipulators (PMs) with $f$-DOF could be commonly described by $f$ possible motion variables on the instantaneous motion space (IMS) and $(6-f)$ restriction motion variables on the instantaneous restriction space (IRS). However, in some situations, PMs moving platform may accompanied by a concomitant (parasitic) motions along the direction of restriction space. Therefore, the commonly understood one-to-one correspondence between joint space and task space mobility of non-redundant PMs would be compromised. This phenomenon occurred due to the fact that the alignment of screws can change the reciprocal screws while the given screw systems are still maintained. To demonstrate the proposed method, an amplitude-based concomitant motion comparison is performed on two widely utilized lower-mobility parallel mechanisms, 3RPS and 3PRS. The result has shown that for the selected mechanisms, concomitant motion is identical regardless of their difference in joint arrangements in each limbs.
\end{abstract}

Keywords: PMs $\cdot$ Parasitic motion $\cdot$ IRS $\cdot$ IMS

\section{Introduction}

The number of actuated joints in most non-redundant PMS at the joint space is considered to be equal to the number of DOF of the end-effector spanned by possible motion vectors [1] and this can be obtained through different mobility calculation methods [2]. Yet, some of the approaches may be able to reveal the existence of some parasitic motions but its source, behavior, and relation to the main motion of the manipulator is challenging to identify. As a result, researchers have conducted several kinematic studies in order to identify and characterize such motions. The research on PMs concomitant motion could be tracked back to the late $90^{\text {th }}$ following the invention of 3RPS mechanism by Hunt [3]. In 2000, Carretero conducted a position level analysis to this class 
of manipulator, and identified the end-effector with three concomitant motions accompanying one transnational and two rotational independent DOF [4]. Lin et al. [5], in 2018, introduced a geometric method to define, classify, shield or use these parasitic motions. In the same year, Huo used conformal geometric algebra (CGA) method [6] to analyze finite motion of PMs with concomitant ones. Previously, Li has made a comparison of parasitic motion among 3PRS mechanisms considering different joint arrangements and showed that the dependency of concomitant motion on different limb arrangement and location of spherical joint [7]. In general, several authors agreed that concomitant motion is an inherent characteristics of parallel manipulators with fewer DOF. Even though, scholars have made great effort to date in describing this motion, it is still an open problem that limits the invention of new mechanisms with the desired motion geometry [6].

\section{Concomitant Motion}

In order to accurately depict the motion of moving platform, understanding the instantaneous restriction space (IRS) and instantaneous motion space (IMS) is essential $[8,11]$. IRS and IMS are said to be complementary subspace to one another that IRS belongs to the no motion space while IMS is for the freedom of moving platform. In other word, IMS is related to DOF of the manipulator. However, unfortunately some fewer DOF manipulators may have extra motion called concomitant movement in the direction of restriction space. Such motion should be taken into account due to its potential effect on the application and intended accuracy of the task. Though, the amplitude of these motion is small [7], it should not be underestimated. If IMS is equal to the number of independent actuators, the system can be stabilized based on the predetermined path, however, the existence of concomitant motion can affect the system stability. Thus, identification of the existence, type, and amplitude of concomitant motion is vital.

\subsection{Basics of Concomitant Motion}

As it is mentioned in Sect. 2, concomitant motion is a strange phenomenon often appeared in the restricted direction of the end-effector of parallel mechanisms. This motion is characterized as unwanted motion that affects the accuracy of the mechanism within the work-space. The good simple example to describe this problem is a four bar linkage shown in Fig. 1. Let the coupler point $o^{\prime}$ of the given four-bar mechanism shown be the center of end-effector. If one wanted to make a linear motion along $x$ or along $y$, it is only possible to get the result with minimal error by doing some parameter optimization. Generally, any point on the coupler induces a combined motion along $\mathrm{x}, \mathrm{y}$ and about $\mathrm{z}$ axis. Thus, The complete orientation of the coupler can be described by $\boldsymbol{p}$ and $\phi$ which clearly shows two dependent motions of the end-effector. Where, $\phi$ is $\theta+\beta$ of a branch. This phenomenon affects the accuracy of the manipulators and some industries 


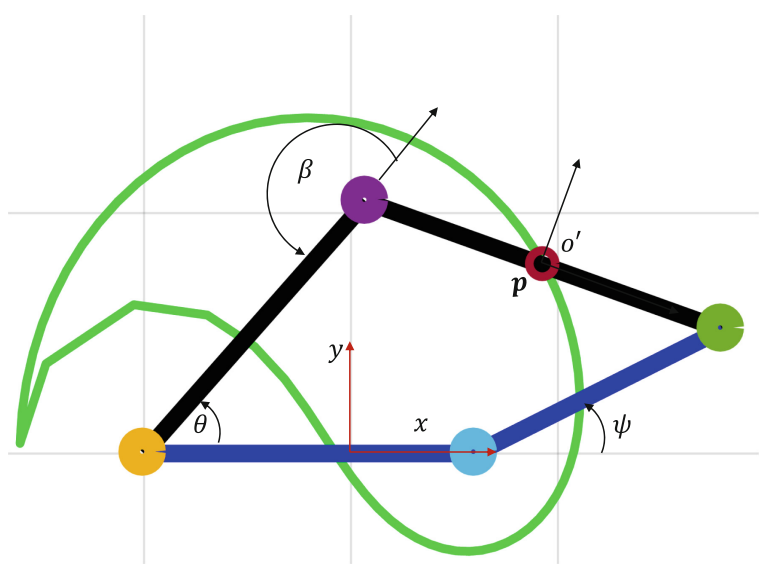

Fig. 1. Four-bar linkage

use external compensation method [13] while others maximize it to utilize [12] this effect for higher DOF operation using lower mobility manipulators.

\section{Velocity Level Constraint Relation to Detect Concomitant Motion}

Since structural constraints may not be fully integrated into the position level equations, velocity level relations can provide additional and a better insight to reveal the concomitant motion phenomenon. The Jacobian of actuation and restriction, feasible motion of the end-effector, and independent variables in the joint space are analyzed. In this study, an analytic method to obtain the two fundamental sub-spaces of the Jacobian is performed based on [9]. Henceforth, equations to describe concomitant motion will be derived based on 3PRS and 3RPS manipulators shown in Fig. 2 (schematics) and Fig. 3 (3D models).

\subsection{Mechanism Description}

Each limb in the manipulator comprises five DOF joints, that are revolute, prismatic, and spherical disregarding the order. Frame $\mathcal{F}$ is attached at the center of base plane and Frame $\mathcal{M}$ is attached at the center of moving platform. $a$ and $b$ are radius of the base and moving platform respectively. $\mathbf{b}_{i}$ is the vector from $\mathbf{p}_{5, i}$ to $\mathcal{M}$ based on $\mathcal{F}$. Spherical joints located at $\mathbf{p}_{5, i}$ connects the leg with moving platform. All three legs of both mechanisms are arranged to be located by $120^{\circ}$ difference around the fixed frame to connect the two plates. $x$ and $y$ axes of $\mathcal{F}$ are in the base plate, $x$ directing along the first leg. Frame 0 is a local or leg coordinate used to derive leg Jacobian and vectors super-scripted with this reference are expressed in the local coordinate. Vectors without superscript 0 are referenced in frame $\mathcal{F}$. $\mathbf{p}_{5 i}$ is the position vector locating the center spherical 

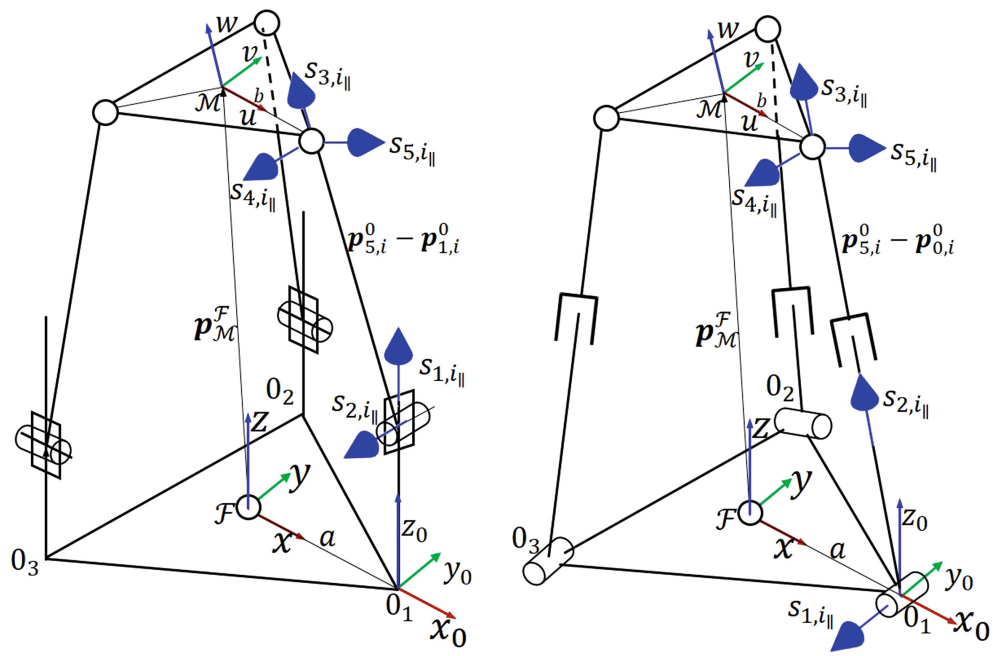

Fig. 2. Schematic diagram. 3PRS (Left), 3RPS (Right)

joint, $\mathbf{p}_{1, i}$ and $\mathbf{p}_{0, i}$ are position of the first joint for the PRS and RPS respectively. $\left(\mathbf{p}_{5, i}-\mathbf{p}_{0, i}\right)$ and $\left(\left(\mathbf{p}_{5, i}-\mathbf{p}_{1, i}\right)\right)$ are vectors of RPS and PRS legs along the rod directed to the center of spherical joint. $\mathbf{p}_{\mathcal{M}}^{\mathcal{F}}$ is the position of center of moving plate with respect to fixed frame.
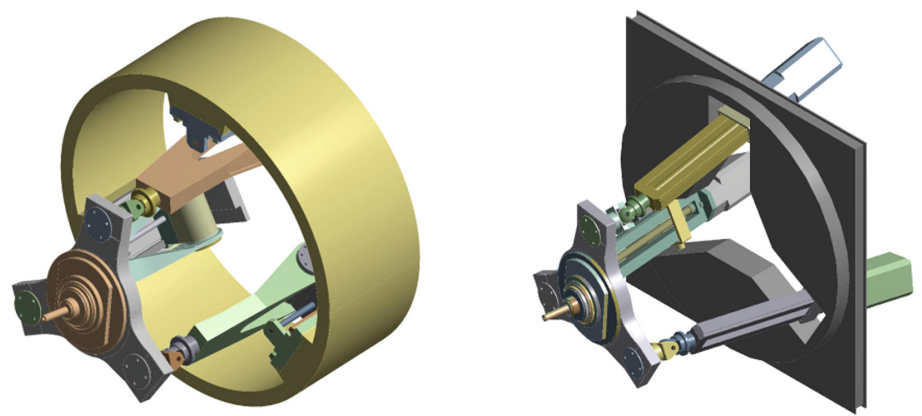

Fig. 3. 3D model. 3PRS (Left), 3RPS (Right)

\subsection{Leg and Manipulator Jacobian}

Once the Jacobian of isolated leg is derived in reference to the local frame 0 , it can be transformed to the fixed and moving platform reference frames by the following transformation matrix.

$$
\mathbf{M}=\left[\begin{array}{cc}
R_{z}\left(\xi_{i}\right) & \mathbf{0} \\
-\left[\mathbf{b}_{i}\right]_{\times} R_{z}\left(\xi_{i}\right) & R_{z}\left(\xi_{i}\right)
\end{array}\right]
$$

where, $\xi_{i}$ is given by $(i-1) \frac{2 \pi}{3}$ for $i=1,2,3$. 
Now, all direction and position vectors are represented in frame $\mathcal{F}$. Then, let $s_{1 i \|}, s_{2 i \|}, s_{3 i \|}, s_{4 i \|}$, and $s_{5 i \|}$ be the direction vectors of isolated legs from base to top plate as shown in Fig. 2 expressed in the fixed frame. Similarly, $s_{1 i_{\perp}}$, $s_{2 i_{\perp}}, s_{3 i_{\perp}}, s_{4 i_{\perp}}$ and $s_{5 i_{\perp}}$ are defined to be the moment vectors. $s_{i_{\|}}^{c}$ is the leg restriction screw imposed on the end-effector by $i^{\text {th }}$ leg. Accordingly, extended Jacobian of the leg is derived with the method proposed in [9].

$$
\begin{aligned}
\mathbf{J}_{e P R S} & =\left[\begin{array}{cccccc}
\mathbf{0} & s_{2 i_{\|}} & s_{3 i_{\|}} & s_{4 i_{\|}} & s_{5 i_{\|}} & \mathbf{0} \\
s_{1 i_{\|}} & s_{2 i_{\perp}} & s_{3 i_{\perp}} & s_{4 i_{\perp}} & s_{5 i_{\perp}} & s_{i_{\|}}^{c}
\end{array}\right] \\
\mathbf{J}_{e R P S} & =\left[\begin{array}{cccccc}
s_{1 i_{\|}} & \mathbf{0} & s_{3 i_{\|}} & s_{4 i_{\|}} & s_{5 i_{\|}} & \mathbf{0} \\
s_{1 i_{\perp}} & s_{2 i_{\|}} & s_{3 i_{\perp}} & s_{4 i_{\perp}} & s_{5 i_{\perp}} & s_{i_{\|}}^{c}
\end{array}\right]
\end{aligned}
$$

Following similar fashion, reciprocal screw relation, inverse Jacobian of the two mechanism can be obtained as

$$
\begin{aligned}
& \mathbf{G}_{P R S}^{T}=\left[\begin{array}{cc}
\left(\mathbf{p}_{5, i}-\mathbf{p}_{1, i}\right)^{T} & \left(\left(\mathbf{p}_{5, i}-\mathbf{p}_{1, i}\right) \times \mathbf{b}_{i}\right)^{T} \\
s_{2 i_{\|}}^{T} & \left(s_{2 i_{\|}} \times \mathbf{b}_{i}\right)^{T}
\end{array}\right] \\
& \mathbf{G}_{R P S}^{T}=\left[\begin{array}{cc}
\left(\mathbf{p}_{5, i}-\mathbf{p}_{0, i}\right)^{T} & \left(\left(\mathbf{p}_{5, i}-\mathbf{p}_{0, i}\right) \times \mathbf{b}_{i}\right)^{T} \\
s_{1 i_{\|}}^{T} & \left(s_{1 i_{\|}} \times \mathbf{b}_{i}\right)^{T}
\end{array}\right]
\end{aligned}
$$

For $i=1,2,3$. With the Jacobian (Inverse) $\mathbf{G}$, the rate kinematics (IRK) can be obtained as follows.

$$
\dot{\boldsymbol{q}}=\mathbf{G}^{T} \$_{t}
$$

Equation 6 comprises the motion and constraint wrench vectors. The more detailed expression holds the following relation.

$$
\left[\begin{array}{c}
\dot{\mathbf{q}}_{a} \\
\dot{\mathbf{q}}_{c}
\end{array}\right]=\left[\begin{array}{ll}
\mathbf{G}_{a v}^{T} & \mathbf{G}_{a \omega}^{T} \\
\mathbf{G}_{c v}^{T} & \mathbf{G}_{c \omega}^{T}
\end{array}\right] \$_{t}
$$

\subsection{Detection of Concomitant Motion}

As far as $\dot{\mathbf{q}}_{c}$ in Eq. 7 is leg constraint, it should always be zero. Therefore, the active joint rate equation must satisfy the following constraint.

$$
\mathbf{G}_{c}^{T} \$_{t}=0
$$

For Eqs. 7 and 8 to meet the above relations requirement, $\$_{t}$ must be a feasible velocity profile for the manipulator.

With the assurance of above constraint relation, we need to obtain independent motions of end-effector. This can be achieved by solving the permitted twist (motion) of the moving plate (PTM) at the home configuration of the manipulator when fixed and moving plates are parallel to each other. i.e.

$$
\mathbf{P T M}=\mathbf{I}-\mathbf{G}_{c} \mathbf{G}_{c}^{W \dagger}
$$


Since $\mathbf{G}_{c}$ is in a ray order, $\mathbf{P T M}$ is in axis order. Equation 9 can be expanded to the $6 \times 6$ matrix as

$$
\mathbf{P T M}=\left[\begin{array}{llllll}
s_{11_{\perp}} & s_{12_{\perp}} & s_{13_{\perp}} & s_{11_{\|}} & s_{12_{\|}} & s_{13_{\|}} \\
s_{21_{\perp}} & s_{22_{\perp}} & s_{23_{\perp}} & s_{21_{\|}} & s_{22_{\|}} & s_{23_{\|}} \\
s_{31_{\perp}} & s_{32_{\perp}} & s_{33_{\perp}} & s_{31_{\|}} & s_{32_{\|}} & s_{33_{\|}} \\
s_{41_{\perp}} & s_{42_{\perp}} & s_{43_{\perp}} & s_{41_{\|}} & s_{42_{\|}} & s_{43_{\|}} \\
s_{51_{\perp}} & s_{52_{\perp}} & s_{53_{\perp}} & s_{51_{\|}} & s_{52_{\|}} & s_{53_{\|}} \\
s_{61_{\perp}} & s_{62_{\perp}} & s_{63_{\perp}} & s_{61_{\|}} & s_{62} & s_{63_{\|}}
\end{array}\right]
$$

at $t=0$, we know all the elements of $\mathbf{G}_{c}$. Solving, PTM gives us

$$
\text { PTM }=\left[\begin{array}{llllll}
0 & 0 & 0 & 0 & 0 & 0 \\
0 & 0 & 0 & 0 & 0 & 0 \\
0 & 0 & 1 & 0 & 0 & 0 \\
0 & 0 & 0 & 1 & 0 & 0 \\
0 & 0 & 0 & 0 & 1 & 0 \\
0 & 0 & 0 & 0 & 0 & 0
\end{array}\right]
$$

Equation 11 tells that the moving plate has one translation motion along the $z$ and two rotations about $x$ and $y$ axis. Therefore, these motions can be fully described by the three independent joint variables.

Now, lets us change the configuration to

$$
\mathbf{d}=\left[\begin{array}{lll}
108.7615 & 114.7896 & 15.9094
\end{array}\right]^{T}
$$

$\mathbf{G}_{c}^{T}$ becomes

$$
\mathbf{G}_{c}^{T}=\left[\begin{array}{cccccc}
0 & -1 & 0 & -0.002 & 0 & -0.1 \\
0.866 & 0.5 & 0 & -0.00305 & 0.0052 & -1 \\
-0.866 & 0.5 & 0 & -0.0048 & -0.0083 & -1
\end{array}\right]
$$

Hence, PTM based on Eq. 9 is

$$
\left[\begin{array}{cccccc}
0.0001 & 0 & 0 & -0.0010 & -0.0078 & 0 \\
0 & 0.0001 & 0 & -0.0078 & 0.0010 & 0.0001 \\
0 & 0 & 1 & 0 & 0 & 0 \\
-0.0010 & -0.0078 & 0 & 0.9999 & 0 & -0.0078 \\
-0.007 & 0.0010 & 0 & 0 & 0.9999 & -0.0010 \\
0 & 0.0001 & 0 & -0.0078 & -0.0010 & 0.0001
\end{array}\right]
$$

Previously, it is known that the manipulator moving plate has three independent motions $(\dot{z}, \dot{\theta}$ and $\dot{\phi})$ correspond to the third, fourth and fifth row of PTM. However, now at this configuration, the moving plate has another three motions in the restricted space, i.e., $\dot{x}, \dot{y}$, and $\dot{\psi}$ corresponding to row one, two and six of PTM. Hence, these newly emerged motions are regarded as concomitant motion of the moving platform. Identifying and quantifying these de-centering motions of the moving plate is quite important in order to utilize or minimizing from the workspace. 


\subsection{Identification of Concomitant Motion}

Identification of the type and direction of these motion is automatic for those who used restriction screw system. Originally, the inverse Jacobian is derived in the ray order and obviously permitted motion of the platform is in the axis order $\left([\boldsymbol{v} \boldsymbol{\omega}]^{T}\right)$. Thus, first second and third row of Eq. 14 are belongs to the permitted transnational motion $\boldsymbol{v}$ of the platform while row four to six belongs to the rotational part. Accordingly, from the type point view the manipulator contain two transnational and one rotational motions. Their direction is also along the restricted axis $x, y$ and $z$, respectively. The overall procedure is outlined as shown in Fig. 4 and summerized as follow.

i. Set the manipulator at home configuration or have the moving and fixed platform parallel.

ii. Derive the constraint wrench.

iii. Get the permitted twist of the moving platform (PTM).

iv. Count the linearly independent rows of PTM, identify their type and directions. These are independent motions of the moving platform.

v. Change manipulator position and orientation.

vi. Again compute the (PTM) matrix in Eq. 9.

vii. Identify the new non-zero rows of PTM matrix.

viii. Determine the type and direction of these new motions at this instant configuration.

ix. Remove independent motion parameters moving plate from PTM matrix. The remaining non-zero variables span the concomitant motion.

Determination of their magnitude will be covered in the Sect. 4 .

\section{Relation of the Parasitic Motion and Independent Motion}

From the previous sections, we know independent motions of the moving plate are

$$
\left[\begin{array}{llllll}
0 & 0 & \dot{z} & \dot{\theta} & \dot{\phi} & 0
\end{array}\right]^{T}
$$

multiplying Eq. 10 with Eq. 15 gives,

$$
\left[\begin{array}{c}
\dot{x} \\
\dot{y} \\
\dot{\psi}
\end{array}\right]=\left[\begin{array}{lll}
s_{13_{\perp}} & s_{11_{\|}} & s_{12} \| \\
s_{23_{\perp}} & s_{21_{\|}} & s_{22_{\|}} \\
s_{63_{\perp}} & s_{61_{\|}} & s_{62}
\end{array}\right]\left[\begin{array}{c}
\dot{z} \\
\dot{\theta} \\
\dot{\phi}
\end{array}\right]
$$

Equation 16 is the relation of concomitant and parasitic motion.

For more generic expression, we can establish the following relation by shifting independent motions to the first three rows and the remaining to the last three rows and transform the matrix to upper block triangular matrix.

$$
\left[\begin{array}{c}
\mathbf{s}_{t a I} \\
\mathbf{s}_{t a D}
\end{array}\right]=\left[\begin{array}{cc}
\mathbf{M}_{I v}^{e} & \mathbf{M}_{I \omega}^{e} \\
\mathbf{0} & \mathbf{M}_{D \omega}^{e}
\end{array}\right]\left[\begin{array}{c}
\mathbf{s}_{t I} \\
\mathbf{s}_{t D}
\end{array}\right]
$$




$$
\begin{gathered}
\mathbf{s}_{t a I}=\left[\begin{array}{lll}
\dot{z}_{a} & \dot{\theta}_{a} & \dot{\phi}_{a}
\end{array}\right]^{T} \\
\mathbf{s}_{t a D}\left[\begin{array}{lll}
\dot{x}_{a} & \dot{y}_{a} & \dot{\psi}_{a}
\end{array}\right]^{T}
\end{gathered}
$$

In Eqs. 18 and $19 \mathbf{s}_{t a I}$ and $\mathbf{s}_{t a D}$ are independent and dependent twist of the moving plate. $\mathbf{M}_{I v}^{e}$ is a $3 \times 3$ matrix spanned by independent linear twist of the moving plate, $\mathbf{M}_{I \omega}^{e}$ is $3 \times 3$ matrix spanned by independent rotational twist of the moving plate while $\mathbf{M}_{D \omega}^{e}$ is another $3 \times 3$ matrix spanned by dependent rotational motion of the moving plate. On the left side of Eq. 17, the arbitrarily given independent and dependent motions of the moving plate are shown.

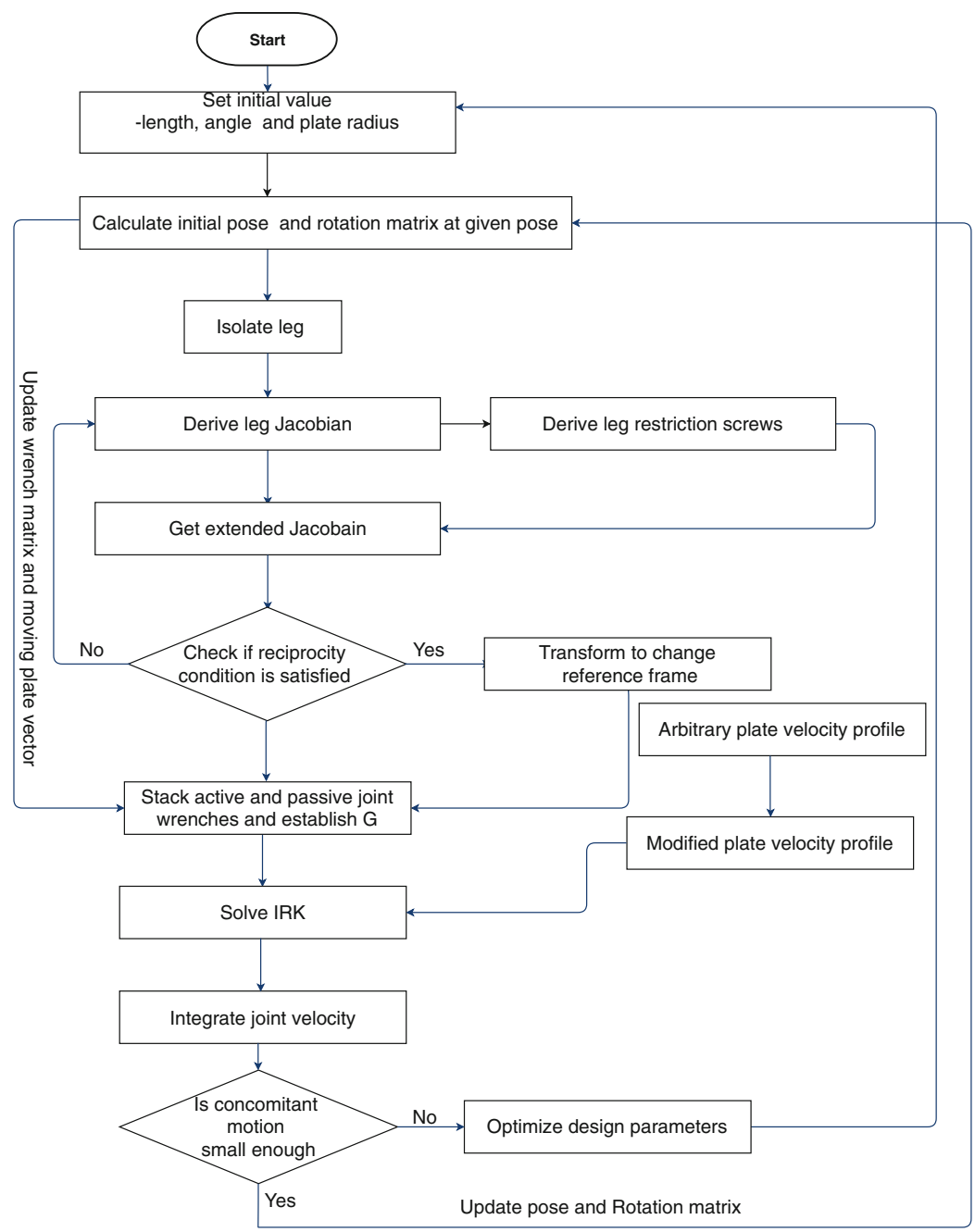

Fig. 4. The general outline of the analysis of concomitant motions 
The upper block triangular matrix that maps the feasible and arbitrary motion of the moving plate is transformed projection matrix. Rearranging Eq. 17 yields

$$
\mathbf{s}_{t a D}=\mathbf{M}_{D \omega}^{e}\left(\mathbf{M}_{I \omega}^{e \dagger}\left(\mathbf{s}_{t a I}-\mathbf{M}_{I v}^{e} \mathbf{s}_{t I}\right)\right)
$$

In 20, $\mathbf{M}_{I \omega}^{e \dagger}$ is a moore-penrose pseudo inverse of $\mathbf{M}_{I \omega}^{e}$. By integrating $\mathbf{s}_{t a D}$ we can get the undesired motion of the moving plate (concomitant motion).

Another option to derive the relation between concomitant and main motion of the moving platform is using the position level relation. Referring to Fig. 2, the constraint equation at the position level is exploited. Let $\mathbf{R}$ be the rotation matrix and $\mathbf{b}_{i}$ the vector directed from center of moving plate to the center of spherical joint. Referring to Fig. 2, the position of spherical joints can be described by

$$
\mathbf{p}_{5, i}=\mathbf{p}_{\mathcal{M}}^{\mathcal{F}}+\mathbf{b}_{i}
$$

where, $\mathbf{b}_{i}=\mathbf{R} \mathbf{b}_{i}^{\prime}$. Hence, Expanded from of Eq. 21 is

$$
\begin{gathered}
\mathbf{p}_{5,1}=\left[\begin{array}{l}
x+b r_{11} \\
y+b r_{21} \\
z+b r_{31}
\end{array}\right] \\
\mathbf{p}_{5,2}=\left[\begin{array}{l}
x-\frac{1}{2} r_{11} b+\frac{\sqrt{3}}{2} b r_{12} \\
y-\frac{1}{2} r_{21} b+\frac{\sqrt{3}}{2} b r_{22} \\
z-\frac{1}{2} r_{31} b+\frac{\sqrt{3}}{2} b r_{32}
\end{array}\right] \\
\mathbf{p}_{5,3}=\left[\begin{array}{l}
x-\frac{1}{2} r_{11} b-\frac{\sqrt{3}}{2} b r_{12} \\
y-\frac{1}{2} r_{21} b-\frac{\sqrt{3}}{2} b r_{22} \\
z-\frac{1}{2} r_{31} b-\frac{\sqrt{3}}{2} b r_{32}
\end{array}\right]
\end{gathered}
$$

Due to revolute joint the following position level constraint is imposed on the motion of spherical joint.

$$
\mathbf{p}_{5, i}^{T} s_{2 i \|}=0
$$

where, $s_{j i \|}$ is the direction vector of the $1^{\text {st }}\left(2^{\text {nd }}\right)$ joint of RPS(PRS) mechanisms.

$$
p_{51 y}=0, p_{52 y}=-\sqrt{3} p_{52 x}, p_{53 y}=\sqrt{3} p_{53 x}
$$

Substitute from Eqs. 22 to 24 into 25 gives,

$$
\begin{gathered}
x=-\frac{1}{2} b\left(r_{22}-r_{11}\right) \\
y=\frac{1}{2}\left(r_{21}-3 r_{12}\right)
\end{gathered}
$$

Equations 27 and 28 are the concomitant motion of moving platform as a function of two independent rotational parameters. By taking differentiation of these equations with respect to independent variables contained in it, we can obtain their velocity level relation. Figure 5 shows the leg displacement that 
resulted the workspace of the platform including the parasitic motion. Figure 6 shows the comparison of the two mechanisms based on the amplitude of concomitant motion generated by the moving plate center due to the respected leg displacements. As the result indicates, 3PRS and 3RPS manipulator has the same concomitant (dependent) motion both in direction and amplitude within the workspace. Geometric parameters of the two mechanisms used in the implementation of the proposed approach and the numerical simulation are given in Table 1.

Table 1. Manipulator parameters

\begin{tabular}{l|l|l|l|l|l}
\hline PKMs & $a$ & $b$ & $l$ & $\theta$ in deg & $\psi$ in deg \\
\hline PRS & $350 \mathrm{~mm}$ & $250 \mathrm{~mm}$ & $657.6473 \mathrm{~mm}$ & {$[-50,-50]$} & {$[0,360]$} \\
\hline RPS & $350 \mathrm{~mm}$ & $250 \mathrm{~mm}$ & $657.6473 \mathrm{~mm}$ & {$[-50,-50]$} & {$[0,360]$} \\
\hline
\end{tabular}

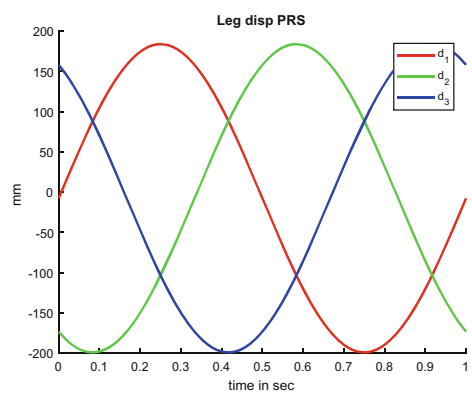

(a) 3PRS

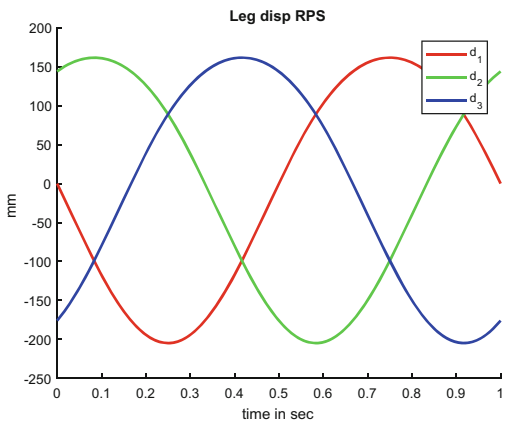

(b) 3PRS

Fig. 5. Leg displacements

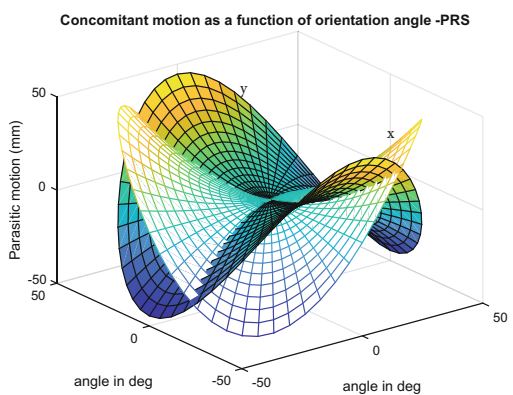

(a) 3PRS

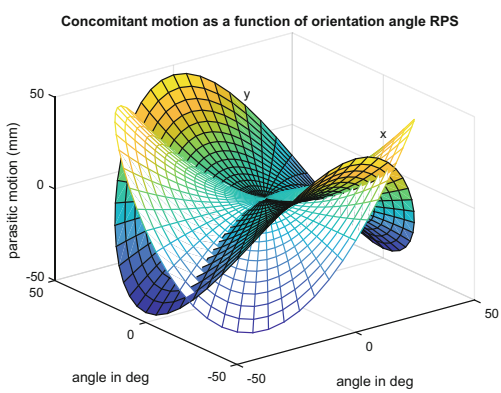

(b) 3RPS

Fig. 6. Concomitant motion in the work space 
The rotation matrix used to induce orientation workspace is based on T-T angle presented in [10]. Since these mechanisms are analyzed as a parallel kinematic machines (PKMs), the set of all feasible pairs of azimuth and tilt angles are used to define the orientation leaving the torsion angle zero.

\section{Conclusion}

On the basis of velocity level constraint relation, concomitant motion of lower mobility parallel manipulators is studied. The method of detection and identification of these motion of the moving platform is shown in a very simple and intuitive manner using existing constraint Jacobian without any extra effort of derivation. As a result, the type, direction and amplitude of concomitant motion is identified to be independent on their joint arrangements in limbs.

\section{References}

1. Tsai L-W (1999) Robot Analysis and Design: The Mechanics of Serial and Parallel Manipulators, 1st edn. John Wiley and Sons Inc, New York

2. Briot S, Khalil W (2015) Dynamics of Parallel Robots, vol 35, September 2015. http://www.rsc.org/dose/titleofsubordinatedocument. Accessed 15 Jan 1999

3. Hunt KH (1983) Structural kinematics of in-parallel-actuated robotarms. J Mech Trans Autom Des 105(4):705-712. https://doi.org/10.1115

4. Carretero JA, Podhorodeski RP, Nahon MA, Gosselin CM (1999) Kinematic analysis and optimization of a new three degree of freedom spatial parallel manipulator. J Mech Des 122(1):1724. https://doi.org/10.1115/1.533542

5. On Parasitic Motion of Parallel Mechanisms, ser. (2016) International Design Engineering Technical Conferences and Computers and Information in Engineering Conference, vol Volume 5B: 40th Mechanisms and Robotics Conference, v05BT07A077, August 2016. https://doi.org/10.1115/DETC2016-59859

6. Huo X, Song Y (2018) Finite motion analysis of parallel mechanisms with parasitic motions based on conformal geometric algebra. Adv Appl Clifford Algebras 28(1):21. https://doi.org/10.1007/s00006-018-0832-4

7. Li Q, Chen Z, Chen Q, Wu C, Hu X (2011) Parasitic motion comparison of 3PRS parallel mechanism with different limb arrangements. Robot Comput-Integr Manuf 27(2):389-396. Translational Research Where Engineering Meets Medicine. http://www.sciencedirect.com/science/article/pii/S0736584510001067

8. Kim D, Chung WK (2003) Kinematic condition analysis of three- DOF pure translational parallel manipulators. J Mech Des 125(2):323

9. Analytic formulation of reciprocal screws and its application to nonredundant robot manipulators. J Mech Des 125(1):158-164. https://doi.org/10.1115/1.1539508

10. Bonev IA (2002) Geometric analysis of parallel mechanisms [Dissertation]. Laval University, Quebec, Canada

11. Nigatu H, Singh AP, Solomon Seid M (2013) Acceleration analysis of 3DOF parallel manipulators. Glob J Res Eng 12(7-A). https://engineeringresearch.org/index. php/GJRE/article/view/689

12. Huang Z, Chen Z, Liu J, Liu S (2011) A 3DOF rotational parallel manipulator without intersecting axes. J Mech Robot 3(2):201

13. Zadoks R, Midha A (1987) Parametric stability of a two-degree-of-freedom machine system: part I-equations of motion and stability. J Mech Transmissions Autom Des 109(2):210-215. https://doi.org/10.1115/1.3267440 